\title{
Convergence of powers and Canonical form of s-transitive intuitionistic fuzzy matrix
}

\author{
Riyaz Ahmad Padder and P. Murugadas \\ Department of Mathematics Annamalai University, India
}

Received: 5 May 2016, Accepted: 18 August 2016

Published online: 16 August 2017.

\begin{abstract}
In this paper various properties of s-transitive intuitionistic fuzzy matrices are discussed. We obtain some results regarding convergence of powers of s-transitive intuitionistic fuzzy matrices. By using the properties of s-transitive intuitionistic fuzzy matrices we formulated and constructed canonical form.
\end{abstract}

Keywords: Intuitionistic fuzzy set, intuitionistic fuzzy matrix, s-transitive, convergence powers of intuitionistic fuzzy matrix, canonical form.

\section{Introduction}

The theory of fuzzy sets is used in various Mathematical fields. Zadeh [1] 1965 developed the concept of fuzzy sets which is the basis of fuzzy Mathematics. Since then various researchers worked on the development of fuzzy set theory. Atanassov [2,3,4,5,6,7] has given idea about intuitionistic fuzzy sets. Im and Lee [8] studied about the determinant of square intuitionistic fuzzy matrices (IFMs). Pal et.al [9] discussed (IFMs). Pal and Shyamal [10] defined distance between (IFMs). Bhowmik and Pal [11,12] discussed few properties of (IFMs), intuitionistic circulant fuzzy matrices and generalized (IFMs). Meenakhsi and Gandhimathi [13] developed intuitionistic fuzzy relational equations. Sriram and Murugadas [14,15] developed the concept of semiring and sub-inverse of (IFMs). Murugadas and Lalitha [16, 17, 18] applied implication operators and defined sub-inverse, g-inverse and decomposition of (IFMs). The authors [19] have studied reduction of rectangular (IFM). The theory of IFM is very important for the study of intuitionistic fuzzy relations. Thomason [20] studied about the convergence powers of fuzzy matrix. He provided the sufficient condition for convergence of fuzzy matrix. Buckley [21] Ran and Liu [22] and Gregory et al. [23] after using max-min operation of fuzzy matrix obtained only two results, either the fuzzy matrix convergences to idempotent matrices or oscillates to finite period. Hashimoto [24] explored the convergence of the power of a fuzzy transitive matrix. Lur et al. [25] studied about convergence of powers for a fuzzy matrix by using maxmin and max-arithmetic mean operations. Kolodziejczyk [26] discussed convergence of powers of s-transitive fuzzy matrix. Xin [27] studied the convergence of powers of controllable fuzzy matrix. He also showed that controllable fuzzy matrix oscillate with period equal 2. Nola [28] worked on the convergence of powers of reciprocal fuzzy matrices and deduced some properties. Kolodziejczyk [29] examined canonical form of s-transitive fuzzy matrix by using max-min transitive fuzzy matrix. Chenggong [30] discussed canonical form of the s-transitive matrices over lattices. Hashimoto [31] studied canonical form of the transitive fuzzy matrix. He reduced a transitive fuzzy matrix into the sum of a nilpotent fuzzy matrix and a symmetric fuzzy matrix. Lee and Jeong [32] studied some properties of canonical form of transitive IFM. An interesting problem in the theory of IFM is the convergence of the powers and canonical form of s-transitive IFM. Many authors worked on this problem. The purpose of this paper is to discuss the convergence of the powers and canonical form of the IFM. 


\section{Definitions}

Definition 1. [2] An Intuitionistic Fuzzy Set (IFS) A in X (universal set) is defined as an object of the following form $A=\left\{\left\langle x, \mu_{A}(x), v_{A}(x)\right\rangle / x \in X\right\}$, where the functions: $\mu_{A}: X \rightarrow[0,1]$ and $v_{A}: X \rightarrow[0,1]$ define the membership function and non-membership function of the element $x \in X$ respectively and for every $x \in X: 0 \leq \mu_{A}(x)+v_{A}(x) \leq 1$.

Atanassov introduced operations $\left\langle x, x^{\prime}\right\rangle \vee\left\langle y, y^{\prime}\right\rangle=\left\langle\max \{x, y\}, \min \left\{x^{\prime}, y^{\prime}\right\}\right\rangle$ and $\left\langle x, x^{\prime}\right\rangle \wedge\left\langle y, y^{\prime}\right\rangle=\left\langle\min \{x, y\}, \max \left\{x^{\prime}, y^{\prime}\right\}\right\rangle$. Moreover, the operation $\left\langle x, x^{\prime}\right\rangle-\left\langle y, y^{\prime}\right\rangle$ defined by

$$
\left\langle x, x^{\prime}\right\rangle-\left\langle y, y^{\prime}\right\rangle= \begin{cases}\left\langle x, x^{\prime}\right\rangle & \text { if }\left\langle x, x^{\prime}\right\rangle>\left\langle y, y^{\prime}\right\rangle \\ \langle 0,1\rangle & \text { if }\left\langle x, x^{\prime}\right\rangle \leq\left\langle y, y^{\prime}\right\rangle\end{cases}
$$

Definition 2. [33] Let $X=\left\{x_{1}, x_{2}, \ldots x_{m}\right\}$ be a set of alternatives and $Y=\left\{y_{1}, y_{2}, \ldots y_{n}\right\}$ be the attribute set of each element of $X$. An Intuitionistic Fuzzy Matrix (IFM) is defined by $A=\left(\left\langle\left(x_{i}, y_{j}\right), \mu_{A}\left(x_{i}, y_{j}\right), v_{A}\left(x_{i}, y_{j}\right)\right\rangle\right)$ for $i=1,2 \ldots m$ and $j=1,2, \ldots n$, where $\mu_{A}: X \times Y \rightarrow[0,1]$ and $v_{A}: X \times Y \rightarrow[0,1]$ satisfy the condition $0 \leq \mu_{A}\left(x_{i}, y_{j}\right)+v_{A}\left(x_{i}, y_{j}\right) \leq 1$. For simplicity we denote an intuitionistic fuzzy matrix (IFM) as a matrix of pairs $A=\left(\left\langle a_{i j}, a_{i j}^{\prime}\right\rangle\right)$ of a non negative real numbers satisfying $a_{i j}+a_{i j}^{\prime} \leq 1$ for all $i, j$. We denote the set of all IFM of order $m \times n$ by $\mathscr{F}_{m n}$.

For $n \times n$ IFMs $Q=\left(\left\langle q_{i j}, q_{i j}^{\prime}\right\rangle\right)$ and $S=\left(\left\langle s_{i j}, s_{i j}^{\prime}\right\rangle\right)$ with their elements having values in the unit interval $[0,1]$, the following notations are well known:

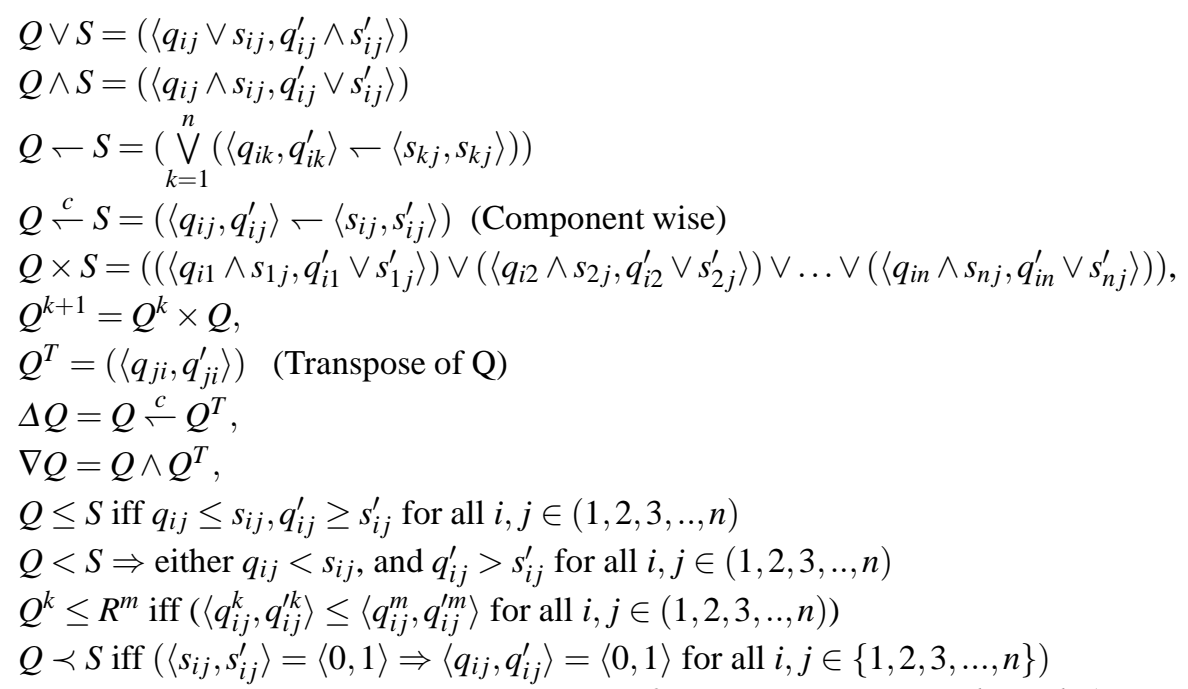

The IFM $\mathrm{Q}$ is called max-min transitive if $Q^{2} \leq Q$, convergent if $Q^{k}=Q^{k+1}$ for some positive integer k, symmetric if $Q=Q^{T}$, idempotent if $Q^{2}=Q$, and nilpotent if $Q^{n}=(\langle 0,1\rangle)$. IFM Q is said to be s-transitive iff $\left\langle q_{i k}, q_{i k}^{\prime}\right\rangle>\left\langle q_{k i}, q_{k i}^{\prime}\right\rangle$ and $\left\langle q_{k j}, q_{k j}^{\prime}\right\rangle>\left\langle q_{j k}, q_{j k}^{\prime}\right\rangle \Rightarrow\left\langle q_{i j}, q_{i j}^{\prime}\right\rangle>\left\langle q_{j i}, q_{j i}^{\prime}\right\rangle$ for any indexes $i, j, k \in\{1,2,3 \ldots . . n\}$, such that $i \neq j, j \neq k, i \neq k$.

Remark. For any IFM $Q=\left(\left\langle q_{i j}, q_{i j}^{\prime}\right\rangle\right)$ it is easily seen that $\Delta(\Delta Q)=\Delta Q$.

Remark. For any IFM $Q=\left(\left\langle q_{i j}, q_{i j}^{\prime}\right\rangle\right)$ it is easily seen that $\nabla Q$ is symmetric.

Theorem 1. [32] If $Q=\left(\left\langle q_{i j}, q_{i j}^{\prime}\right\rangle\right)$ any IFM then $Q=\Delta Q \vee \nabla Q$

Theorem 2. [32] Let $N$ and $S$ be a nilpotent and symmetric IFMs, respectively. Then there exists a permutation matrix $P$ such that $T=\left(\left\langle t_{i j}, t_{i j}^{\prime}\right\rangle\right)=P \times(N \vee S) \times P^{T}$ satisfies $\left\langle t_{i j}, t_{i j}^{\prime}\right\rangle \geq\left\langle t_{j i}, t_{j i}^{\prime}\right\rangle$ for $i>j$.

Theorem 3. [32] For a transitive IFM $Q$, there exists a permutation matrix $P$ such that $T=\left(\left\langle t_{i j}, t_{i j}^{\prime}\right\rangle\right)=P \times Q \times P^{T}$ satisfies $\left\langle t_{i j}, t_{i j}^{\prime}\right\rangle \geq\left\langle t_{j i}, t_{j i}^{\prime}\right\rangle$ for $i>j$. 


\section{Results}

Theorem 4. If $Q=\left(\left\langle q_{i j}, q_{i j}^{\prime}\right\rangle\right)$ is max-min transitive IFM then $Q=\left(\left\langle q_{i j}, q_{i j}^{\prime}\right\rangle\right)$ is s-transitive IFM.

Proof. Let Q be max-min transitive IFM. Assume, $\left\langle q_{i k}, q_{i k}^{\prime}\right\rangle>\left\langle q_{k i}, q_{k i}^{\prime}\right\rangle$ and $\left\langle q_{j i}, q_{j i}^{\prime}\right\rangle>\left\langle q_{i j}, q_{i j}^{\prime}\right\rangle$ then $\left\langle q_{j k}, q_{j k}^{\prime}\right\rangle>\left\langle q_{k j}, q_{k j}^{\prime}\right\rangle$. Suppose if $\left\langle q_{j k}, q_{j k}^{\prime}\right\rangle \leq\left\langle q_{k j}, q_{k j}^{\prime}\right\rangle$. By transitivity of Q, we have $\left\langle q_{j k}, q_{j k}^{\prime}\right\rangle \geq\left\langle q_{j i}, q_{j i}^{\prime}\right\rangle \wedge\left\langle q_{i k}, q_{i k}^{\prime}\right\rangle>\left\langle q_{i j}, q_{i j}^{\prime}\right\rangle \wedge\left\langle q_{k i}, q_{k i}^{\prime}\right\rangle \quad$ (by hypothesis) $\Rightarrow\left\langle q_{j k}, q_{j k}^{\prime}\right\rangle \geq\left\langle q_{k j}, q_{k j}^{\prime}\right\rangle \wedge\left\langle q_{j i}, q_{j i}^{\prime}\right\rangle \wedge\left\langle q_{i j}, q_{i j}^{\prime}\right\rangle=\left\langle q_{k j}, q_{k j}^{\prime}\right\rangle \wedge\left\langle q_{i j}, q_{i j}^{\prime}\right\rangle \Rightarrow\left\langle q_{k j}, q_{k j}^{\prime}\right\rangle>\left\langle q_{i j}, q_{i j}^{\prime}\right\rangle$, on the other hand, $\left\langle q_{i k}, q_{i k}^{\prime}\right\rangle>\left\langle q_{k i}, q_{k i}^{\prime}\right\rangle \geq\left\langle q_{k j}, q_{k j}^{\prime}\right\rangle \wedge\left\langle q_{i j}, q_{i j}^{\prime}\right\rangle$. Since $\left\langle q_{k j}, q_{k j}^{\prime}\right\rangle>\left\langle q_{i j}, q_{i j}^{\prime}\right\rangle \Rightarrow\left\langle q_{i k}, q_{i k}^{\prime}\right\rangle \wedge\left\langle q_{k j}, q_{k j}^{\prime}\right\rangle>\left\langle q_{i j}, q_{i j}^{\prime}\right\rangle$, which is a contradiction to the fact that $\mathrm{Q}$ is max-min transitive. Hence $\left.\left\langle q_{j k}, q_{j k}^{\prime}\right\rangle\right\rangle\left\langle q_{k j}, q_{k j}^{\prime}\right\rangle$.

Theorem 5. If $Q=\left(\left\langle q_{i j}, q_{i j}^{\prime}\right\rangle\right)$ is max-min transitive IFM, then $\Delta Q=Q \stackrel{c}{\leftarrow} Q^{T}$ is max-min transitive IFM.

Proof. Let $S=\left(\left\langle s_{i j}, s_{i j}^{\prime}\right\rangle\right)=\Delta Q=Q \stackrel{c}{\leftarrow} Q^{T}$. Then $\left\langle s_{i j}, s_{i j}^{\prime}\right\rangle=\left\langle q_{i j}, q_{i j}^{\prime}\right\rangle \stackrel{c}{\leftarrow}\left\langle q_{j i}, q_{j i}^{\prime}\right\rangle$. Consider that $\left\langle s_{i k}, s_{i k}^{\prime}\right\rangle \wedge\left\langle s_{k j}, s_{k j}^{\prime}\right\rangle=\left\langle c, c^{\prime}\right\rangle>\langle 0,1\rangle$. We get $\Rightarrow\left\langle q_{i k}, q_{i k}^{\prime}\right\rangle \wedge\left\langle q_{k j}, q_{k j}^{\prime}\right\rangle=\left\langle c, c^{\prime}\right\rangle>\langle 0,1\rangle$ and $\left\langle q_{i j}, q_{i j}^{\prime}\right\rangle \geq\left\langle c, c^{\prime}\right\rangle$.

We show that if $\left\langle q_{j i}, q_{j i}^{\prime}\right\rangle \geq\left\langle q_{i j}, q_{i j}^{\prime}\right\rangle$, then there exists a contradiction.

(i) If $\left\langle q_{i k}, q_{i k}^{\prime}\right\rangle=c$ then $\left\langle q_{k i}, q_{k i}^{\prime}\right\rangle\left\langle\left\langle c, c^{\prime}\right\rangle\right.$. Since $\left\langle q_{k i}, q_{k i}^{\prime}\right\rangle \geq\left\langle q_{k j}, q_{k j}^{\prime}\right\rangle \wedge\left\langle q_{j i}, q_{j i}^{\prime}\right\rangle$. This contradicts with fact of transitivity.

(ii) If $\left\langle q_{k j}, q_{k j}^{\prime}\right\rangle=\left\langle c, c^{\prime}\right\rangle$ then $\left\langle q_{j k}, q_{j k}^{\prime}\right\rangle\left\langle\left\langle c, c^{\prime}\right\rangle\right.$. Since $\left\langle q_{j k}, q_{j k}^{\prime}\right\rangle \geq\left\langle q_{j i}, q_{j i}^{\prime}\right\rangle \wedge\left\langle q_{i k}, q_{i k}^{\prime}\right\rangle$, again a contradiction. Thus $\left\langle s_{i j}, s_{i j}^{\prime}\right\rangle=\left\langle q_{i j}, q_{i j}^{\prime}\right\rangle \geq\left\langle c, c^{\prime}\right\rangle$.

Theorem 6. $Q$ is s-transitive IFM iff $(\Delta Q)^{2} \prec \Delta Q$.

Proof. Let Q be an s-transitive IFM and $\left\langle q_{k h}^{\Delta}, q_{k h}^{\prime \Delta}\right\rangle=\langle 0,1 t\rangle$ for a few $k, h \in\{1,2,3, \ldots n\}$, where $\Delta Q=\left(\left\langle q_{i j}^{\Delta}, q_{i j}^{\prime \Delta}\right\rangle\right)$. Then we have to show that $\max \left\{\left\langle q_{k i}^{\Delta}, q_{k i}^{\prime \Delta}\right\rangle \wedge\left\langle q_{i h}^{\Delta}, q_{i h}^{\prime \Delta}\right\rangle\right\}=\langle 0,1\rangle$. Let us assume that, $\left\langle q_{k j}^{\Delta}, q_{k j}^{\prime \Delta}\right\rangle \wedge\left\langle q_{j h}^{\Delta}, q_{j h}^{\prime \Delta}\right\rangle>\langle 0,1\rangle$ for a few $j \in\{1,2,3 \ldots, n\}$. Hence $\left\langle q_{k j}, q_{k j}^{\prime}\right\rangle>\left\langle q_{j k}, q_{j k}^{\prime}\right\rangle$ and $\left\langle q_{j h}, q_{j h}^{\prime}\right\rangle>\left\langle q_{h j}, q_{h j}^{\prime}\right\rangle$. Thus, by applying the properties of s-transitive IFM of Q, we get $\left\langle q_{k h}, q_{k h}^{\prime}\right\rangle>\left\langle q_{h k}, q_{h k}^{\prime}\right\rangle$ and $\left\langle q_{k h}^{\Delta}, q_{k h}^{\prime \Delta}\right\rangle>\langle 0,1\rangle$. This contradicts with give condition.

Conversely, let $(\Delta Q)^{2} \prec \Delta Q$. Let assume that property of s-transitive IFM does not hold, such that there exists integers $i, k, j \in\{1,2,3 \ldots, n\}$ so that $\left\langle q_{i k}, q_{i k}^{\prime}\right\rangle>\left\langle q_{k i}, q_{k i}^{\prime}\right\rangle,\left\langle q_{k j}, q_{k j}^{\prime}\right\rangle>\left\langle q_{j k}, q_{j k}^{\prime}\right\rangle$ and $\left\langle q_{i j}, q_{i j}^{\prime}\right\rangle \leq\left\langle q_{j i}, q_{j i}^{\prime}\right\rangle$.

Hence, $\left\langle q_{i k}^{\Delta}, q_{i k}^{\Delta \Delta}\right\rangle>\langle 0,1\rangle,\left\langle q_{k j}^{\Delta}, q_{k j}^{\Delta \Delta}\right\rangle>\langle 0,1\rangle$ and $\max \left\{\left\langle q_{i h}^{\Delta}, q_{i h}^{\prime \Delta}\right\rangle \wedge\left\langle q_{h j}^{\Delta}, q_{h j}^{\prime \Delta}\right\rangle\right\} \geq\left\langle q_{i k}^{\Delta}, q_{i k}^{\prime \Delta}\right\rangle \wedge\left\langle q_{k j}^{\Delta}, q_{k j}^{\prime \Delta}\right\rangle>\langle 0,1\rangle$. This means that element which lies in the $(\mathrm{i}, \mathrm{j})$ entry of the matrix $(\Delta Q)^{2}$ is positive, whereas $\left\langle q_{i j}^{\Delta}, q_{i j}^{\prime \Delta}\right\rangle=\langle 0,1\rangle$. This contradicts with the definition of s-transitive intuitionistic fuzzy relation.

\section{Theorem 7. If $Q$ is s-transitive IFM of order $n$ then}

(i) $\Delta Q$ is s-transitive IFM.

(ii) $\triangle Q$ is nilpotent IFM.

Proof. (i) From Theorem 6 and Remark 1 we obtain $(\Delta(\Delta Q))^{2}=(\Delta Q)^{2} \prec \Delta Q=\Delta(\Delta Q)$, implies that $\Delta Q$ is s-transitive.

(ii) Let $(\Delta Q)^{n}=\left(\left\langle q_{i j}^{\Delta, n}, q_{i j}^{\prime \Delta, n}\right\rangle\right)=(\langle 0,1\rangle)$. Suppose if $\Delta Q$ is not nilpotent then there exists indices $i, j \in\{1,2, . . n\}$ so that $\left\langle q_{i j}^{\Delta, n}, q_{i j}^{\Delta, n}\right\rangle>\langle 0,1\rangle$. Then $\left\langle q_{i j}^{\Delta, n}, q_{i j}^{\Delta, n}\right\rangle=\left\langle q_{h_{0} h_{1}}^{\Delta}, q_{h_{0} h_{1}}^{\Delta \Delta}\right\rangle \wedge\left\langle q_{h_{1} h_{2}}^{\Delta}, q_{h_{1} h_{2}}^{\Delta \Delta}\right\rangle \wedge \ldots \wedge\left\langle q_{h_{n-1} h_{n}}^{\Delta}, q_{h_{n-1} h_{n}}^{\Delta}\right\rangle>\langle 0,1\rangle$ for a few integers $h_{0}, h_{1}, h_{2} \ldots, h_{n} \in\{1,2, \ldots, n\}$ so that $h_{0}=i$ and $h_{n}=j$. Then $h_{a}=h_{b}$ for a and $\mathrm{b}(a<b)$ and

$$
\begin{aligned}
& \left\langle q_{h_{a} h_{a+1}}^{\Delta}, q_{h_{a} h_{a+1}}^{\Delta}\right\rangle>\langle 0,1\rangle=\left\langle q_{h_{a+1} h_{a}}^{\Delta}, q_{h_{a+1} h_{a}}^{\Delta}\right\rangle, \\
& \left\langle q_{h_{a+1} h_{a+2}}^{\Delta}, q_{h_{a+1} h_{a+2}}^{\Delta}\right\rangle>\langle 0,1\rangle=\left\langle q_{h_{a+2} h_{a+1}}^{\Delta}, q_{h_{a+2} h_{a+1}}^{\Delta}\right\rangle, \ldots,\left\langle q_{h_{b-1}}^{\Delta} h_{b}, q_{h_{b-1} h_{b}}^{\Delta}\right\rangle>\langle 0,1\rangle=\left\langle q_{h_{b} h_{b-1}}^{\Delta}, q_{h_{b} h_{b-1}}^{\Delta}\right\rangle .
\end{aligned}
$$


By applying the s-transitivity of IFM $\Delta Q$ we get,

$$
\left.\left\langle q_{h_{a} h_{a}}^{\Delta, n}, q_{h_{a} h_{a}}^{\Delta \Delta, n}\right\rangle=\left\langle q_{h_{a} h_{b}}^{\Delta, n}, q_{h_{a} h_{b}}^{\Delta, n}\right\rangle\right\rangle\left\langle q_{h_{b} h_{a}}^{\Delta, n}, q_{h_{b} h_{a}}^{\Delta, n}\right\rangle=\left\langle q_{h_{a} h_{a}}^{\Delta, n}, q_{h_{a} h_{a}}^{\prime \Delta, n}\right\rangle
$$

which is not possible.

Example 1. The following example illustrates that any max-min IFM is s-transitive IFM but the converse is not true.

$$
\begin{aligned}
& Q=\left(\begin{array}{c}
\langle 1.0,0.0\rangle\langle 0.7,0.2\rangle\langle 0.8,0.1\rangle \\
\langle 0.4,0.5\rangle\langle 0.0,1.0\rangle\langle 0.5,0.4\rangle \\
\langle 0.1,0.7\rangle\langle 0.9,0.0\rangle\langle 0.0,1.0\rangle
\end{array}\right), Q^{T}=\left(\begin{array}{c}
\langle 1.0,0.0\rangle\langle 0.4,0.5\rangle\langle 0.1,0.7\rangle \\
\langle 0.7,0.2\rangle\langle 0.0,1.0\rangle\langle 0.9,0.0\rangle \\
\langle 0.8,0.1\rangle\langle 0.5,0.4\rangle\langle 0.0,1.0\rangle
\end{array}\right), \Delta Q=Q \stackrel{c}{\leftarrow} Q^{T}
\end{aligned}
$$

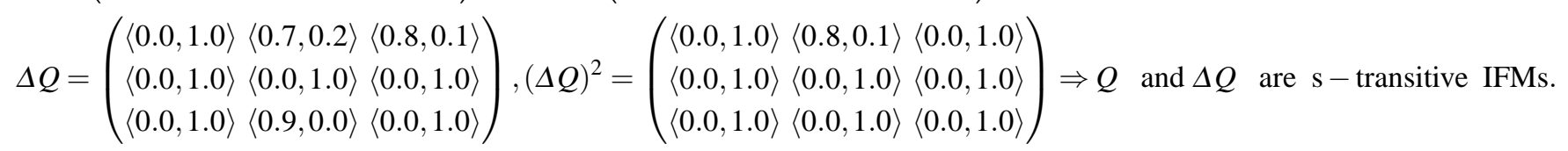

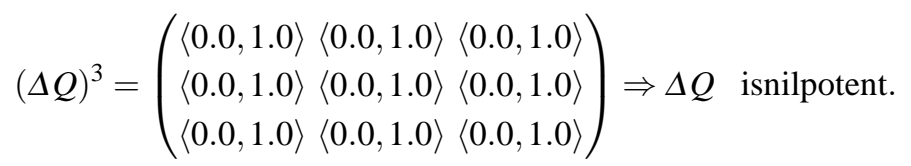

$$
\begin{aligned}
& Q^{2}=\left(\begin{array}{l}
\langle 1.0,0.0\rangle\langle 0.8,0.1\rangle\langle 0.8,0.1\rangle \\
\langle 0.4,0.5\rangle\langle 0.5,0.4\rangle\langle 0.4,0.5\rangle \\
\langle 0.4,0.5\rangle\langle 0.1,0.7\rangle\langle 0.5,0.4\rangle
\end{array}\right) \not \leq Q \Rightarrow Q \text { is not max - min transitive IFM. }
\end{aligned}
$$

Lemma 1. If $Q$ is s-transitive IFM, then

(I) $Q^{n} \leq Q^{n+2} \leq Q^{n+4} \leq Q^{n+6} \ldots$,

(II) $Q^{n+1} \leq Q^{n+3} \leq Q^{n+5} \leq Q^{n+7} \ldots$,

Proof. (I) Let $\left\langle q_{h_{0} h_{n+2 k}}^{n+2 k}, q_{h_{0} h_{n+2 k}}^{\prime n+2 k}\right\rangle=\left\langle q_{h_{0} h_{1}}, q_{h_{0} h_{1}}^{\prime}\right\rangle \wedge\left\langle q_{h_{1} h_{2}}, q_{h_{1} h_{2}}^{\prime}\right\rangle \wedge \ldots \wedge\left\langle q_{h_{n+2 k-1} h_{n+2 k}}, q_{h_{n+2 k-1} h_{n+2 k}}^{\prime}\right\rangle$ for a few $k \in\{0,1,2,3 \ldots\}$. Put $h_{a}=h_{b}$ for some settled $a, b \in\{0,1,2, \ldots n+2 k\}(a<b)$. We have $h_{i} \in\{1,2, \ldots n\}$ for each $i=0,1,2, \ldots, n+2 k$. If $\left\langle q_{h_{i} h_{i+1}}, q_{h_{i} h_{i+1}}^{\prime}\right\rangle>\left\langle q_{h_{i+1} h_{i}}, q_{h_{i+1} h_{i}}^{\prime}\right\rangle$ will hold for every $i=a, a+1, \ldots, b-2, b-1$, then applying the s-transitivity of Q, we get $\left\langle q_{h_{a} h_{a}}, q_{h_{a} h_{a}}^{\prime}\right\rangle=\left\langle q_{h_{a} h_{b}}, q_{h_{a} h_{b}}^{\prime}\right\rangle>\left\langle q_{h_{b} h_{a}}, q_{h_{b} h_{a}}^{\prime}\right\rangle=\left\langle q_{h_{a} h_{a}}, q_{h_{a} h_{a}}^{\prime}\right\rangle$, which is not possible. Thus $\left\langle q_{h_{s} h_{s+1}}, q_{h_{s} h_{s+1}}^{\prime}\right\rangle \leq\left\langle q_{h_{s+1} h_{s}}, q_{h_{s+1} h_{s}}^{\prime}\right\rangle$ for a few $s \in\{a, a+1, \ldots b-1\}$ and

$$
\begin{aligned}
& \left\langle q_{h_{0} h_{n+2 k}}^{n+2 k}, q_{h_{0} h_{n+2 k}}^{\prime n+2 k}\right\rangle=\left\langle q_{h_{0} h_{1}}, q_{h_{0} h_{1}}^{\prime}\right\rangle \wedge\left\langle q_{h_{1} h_{2}}, q_{h_{1} h_{2}}^{\prime}\right\rangle \wedge \ldots \wedge\left\langle q_{h_{s} h_{s+1}}, q_{h_{s} h_{s+1}}^{\prime}\right\rangle \wedge\left\langle q_{h_{s+1} h_{s}}, q_{h_{s+1} h_{s}}^{\prime}\right\rangle \wedge\left\langle q_{h_{s} h_{s+1}}, q_{h_{s} h_{s+1}}^{\prime}\right\rangle \wedge \\
& \left\langle q_{h_{s+1} h_{s+2}}, q_{h_{s+1} h_{s+2}}^{\prime}\right\rangle \wedge \ldots \wedge\left\langle q_{h_{n+2 k-1} h_{n+2 k}}, q_{h_{n+2 k-1} h_{n+2 k}}^{\prime}\right\rangle \Rightarrow\left\langle q_{h_{0} h_{n+2 k}}^{n+2 k}, q_{h_{0} h_{n+2 k}}^{n+2 k}\right\rangle \leq\left\langle q_{h_{0} h_{n+2 k}}^{n+2 k+2}, q_{h_{0} h_{n+2 k}}^{\prime n+2 k+2}\right\rangle
\end{aligned}
$$

put $k=0$ in (3) we get $\left\langle q_{h_{0} h_{n}}^{n}, q_{h_{0} h_{n}}^{\prime n}\right\rangle \leq\left\langle q_{h_{0} h_{n}}^{n+2}, q_{h_{0} h_{n}}^{\prime n+2}\right\rangle \Rightarrow Q^{n} \leq Q^{n+2}$

put $k=1$ in (3) we get $Q^{n+2} \leq Q^{n+4}$

Put $k=2$ in (3) we get $Q^{n+4} \leq Q^{n+6} \Rightarrow Q^{n} \leq Q^{n+2} \leq Q^{n+4} \leq Q^{n+6} \ldots$,

(II) Let $\left\langle q_{h_{0} h_{n+1+2 k}}^{n+1+2 k}, q_{h_{0} h_{n+1+2 k}}^{\prime n+1+2 k}\right\rangle=\left\langle q_{h_{0} h_{1}}, q_{h_{0} h_{1}}^{\prime}\right\rangle \wedge\left\langle q_{h_{1} h_{2}}, q_{h_{1} h_{2}}^{\prime}\right\rangle \wedge \ldots \wedge\left\langle q_{h_{n+1+2 k-1} h_{n+1+2 k}}, q_{h_{n+1+2 k-1} h_{n+1+2 k}}^{\prime}\right\rangle$ for some $k \in\{0,1,2,3 \ldots\}$. Put $h_{a}=h_{b}$ for some settled $a, b \in\{0,1,2, \ldots, n+1+2 k\}(a<b)$. We have $h_{i} \in\{1,2, \ldots n+1\}$ for each $i=0,1,2, \ldots, n+1+2 k$. If $\left\langle q_{h_{i} h_{i+1}}, q_{h_{i} h_{i+1}}^{\prime}\right\rangle>\left\langle q_{h_{i+1} h_{i}}, q_{h_{i+1} h_{i}}^{\prime}\right\rangle$ will hold for every $i=a, a+1, \ldots, b-2, b-1$. Then applying the intuitionistic s-transitivity of Q, we will get $\left.\left\langle q_{h_{a} h_{a}}, q_{h_{a} h_{a}}^{\prime}\right\rangle=\left\langle q_{h_{a} h_{b}}, q_{h_{a} h_{b}}^{\prime}\right\rangle\right\rangle\left\langle q_{h_{b} h_{a}}, q_{h_{b} h_{a}}^{\prime}\right\rangle=\left\langle q_{h_{a} h_{a}}, q_{h_{a} h_{a}}^{\prime}\right\rangle$ 
which is not possible. Thus $\left\langle q_{h_{s} h_{s+1}}, q_{h_{s} h_{s+1}}^{\prime}\right\rangle \leq\left\langle q_{h_{s+1} h_{s}}, q_{h_{s+1} h_{s}}^{\prime}\right\rangle$ for a few $s \in\{a, a+1, \ldots b-1\}$ and

$$
\begin{aligned}
& \left\langle q_{h_{0} h_{n+1+2 k}}^{n+2 k}, q_{h_{0} h_{n+1+2 k}}^{\prime n+1+2 k}\right\rangle=\left\langle q_{h_{0} h_{1}}, q_{h_{0} h_{1}}^{\prime}\right\rangle \wedge\left\langle q_{h_{1} h_{2}}, q_{h_{1} h_{2}}^{\prime}\right\rangle \wedge \ldots \wedge\left\langle q_{h_{s} h_{s+1}}, q_{h_{s} h_{s+1}}^{\prime}\right\rangle \wedge\left\langle q_{h_{s+1} h_{s}}, q_{h_{s+1} h_{s}}^{\prime}\right\rangle \wedge\left\langle q_{h_{s} h_{s+1}}, q_{h_{s} h_{s+1}}^{\prime}\right\rangle \wedge \\
& \left\langle q_{h_{s+1} h_{s+2}}, q_{h_{s+1} h_{s+2}}^{\prime}\right\rangle \wedge \ldots \wedge\left\langle q_{h_{n+1+2 k-1} h_{n+1+2 k}}, q_{h_{n+1+2 k-1} h_{n+1+2 k}}^{\prime}\right\rangle \Rightarrow\left\langle q_{h_{0} h_{n+1+2 k}}^{n+1+2 k}, q_{h_{0} h_{n+1+2 k}}^{n+1+2 k}\right\rangle \leq\left\langle q_{h_{0} h_{n+1+2 k}}^{n+1+2 k+2}, q_{h_{0} h_{n+1+2 k}}^{n+1+2 k+2}\right\rangle
\end{aligned}
$$

put $k=0$ in (5) we get $\left\langle q_{h_{0} h_{n+1}}^{n+1}, q_{h_{0} h_{n+1}}^{\prime n+1}\right\rangle \leq\left\langle q_{h_{0} h_{n}}^{n+3}, q_{h_{0} h_{n}}^{\prime n+3}\right\rangle \Rightarrow Q^{n+1} \leq Q^{n+3}$

put $k=1$ in (5) we get $Q^{n+3} \leq Q^{n+5}$

Put $k=2$ in (5) we get $Q^{n+5} \leq Q^{n+7} \Rightarrow Q^{n+1} \leq Q^{n+3} \leq Q^{n+5} \leq Q^{n+7} \ldots$.

\section{Example 2.}

$$
Q_{1}=\left(\begin{array}{l}
\langle 0.0,1.0\rangle\langle 0.7,0.1\rangle\langle 0.0,1.0\rangle \\
\langle 0.0,1.0\rangle\langle 0.0,1.0\rangle\langle 0.7,0.1\rangle \\
\langle 0.0,1.0\rangle\langle 0.7,0.1\rangle\langle 0.0,1.0\rangle
\end{array}\right), Q_{2}=\left(\begin{array}{c}
\langle 0.0,1.0\rangle\langle 0.7,0.1\rangle\langle 0.0,1.0\rangle \\
\langle 0.0,1.0\rangle\langle 0.0,1.0\rangle\langle 0.7,0.1\rangle \\
\langle 0.7,0.1\rangle\langle 0.0,1.0\rangle\langle 0.0,1.0\rangle
\end{array}\right)
$$

Clearly $Q_{1}$, is s-transitivity IFM but $Q_{2}$ not s-transitivity IFM. One can easily check, $Q_{1}^{3} \neq Q_{1}^{4}, Q_{1}^{3} \not \leq Q_{1}^{4}$, and $Q_{2}^{3} \not \leq Q_{2}^{5}$, $Q_{2}^{4} \not \leq Q_{2}^{6}$. Therefore Lemma 1 is essential for convergence of s-transitive IFM.

\section{Theorem 8. If $Q$ is s-transitive IFM, then}

(i) $Q^{3 n-4}=Q^{3 n-2}$

(ii) $Q^{3 n-3}=Q^{3 n-1}$, for all $n \geq 2$.

Proof. (i) By Lemma 1 it follows that $\left\langle q_{i j}^{3 n-4}, q_{i j}^{13 n-4}\right\rangle \leq\left\langle q_{i j}^{3 n-2}, q_{i j}^{13 n-2}\right\rangle$.

Now we have to show that $\left\langle q_{i j}^{3 n-4}, q_{i j}^{\prime 3 n-4}\right\rangle \geq\left\langle q_{i j}^{3 n-2}, q_{i j}^{\prime 3 n-2}\right\rangle$.

Let

$$
\left\langle q_{i j}^{3 n-2}, q_{i j}^{13 n-2}\right\rangle=\left\langle q_{h_{0} h_{1}}, q_{h_{0} h_{1}}^{\prime}\right\rangle \wedge\left\langle q_{h_{1} h_{2}}, q_{h_{1} h_{2}}^{\prime}\right\rangle \wedge \ldots \wedge\left\langle q_{h_{3 n-3} h_{3 n-2}}, q_{h_{3 n-3} h_{3 n-2}}^{\prime}\right\rangle
$$

where $h_{0}=i$ and $h_{3 n-2}=j$. It is evident that $h_{a}=h_{b}(a<b)$ for few $a, b \in\{0,1,2, . . n\}, h_{c}=h_{d}(c<d)$ for a few $c, d \in\{n-1, n, n+1, \ldots, 2 n-1\}$ and $h_{e}=h_{f}(e<f)$ for some $e, f \in\{2 n-2,2 n-1,2 n, \ldots, 3 n-2\}$. It has been observed that numbers $p=b-a, q=d-c$ and $s=f-e$ are numbers of the elements of the sets

$$
\begin{aligned}
& \left\{\left\langle q_{h_{a} h_{a+1}}, q_{h_{a} h_{a+1}}^{\prime}\right\rangle,\left\langle q_{h_{a+1} h_{a+2}}, q_{h_{a+1} h_{a+2}}^{\prime}\right\rangle, \ldots\left\langle q_{h_{b-1} h_{b}}, q_{h_{b-1} h_{b}}^{\prime}\right\rangle\right\}, \\
& \left\{\left\langle q_{h_{c} h_{c+1}}, q_{h_{c} h_{c+1}}^{\prime}\right\rangle,\left\langle q_{h_{c+1} h_{c+2}}, q_{h_{c+1} h_{c+2}}^{\prime}\right\rangle, \ldots,\left\langle q_{h_{d-1} h_{d}}, q_{h_{d-1} h_{d}}^{\prime}\right\rangle\right\} \text { and } \\
& \left\{\left\langle q_{h_{e} h_{e+1}}, q_{h_{e} h_{e+1}}^{\prime}\right\rangle,\left\langle q_{h_{e+1} h_{e+2}}, q_{h_{e+1} h_{e+2}}^{\prime}\right\rangle, \ldots,\left\langle q_{h_{f-1} h_{f}}, q_{h_{f-1} h_{f}}^{\prime}\right\rangle\right\}
\end{aligned}
$$

correspondingly. Two cases arises.

Case (i) Among $\mathrm{p}, \mathrm{q}$ and $\mathrm{s}$ at least one number should be even number say (p). Eliminating $\left\langle q_{h_{a} h_{a+1}}, q_{h_{a} h_{a+1}}^{\prime}\right\rangle \wedge\left\langle q_{h_{a+1} h_{a+2}}, q_{h_{a+1} h_{a+2}}^{\prime}\right\rangle \wedge, \ldots, \wedge\left\langle q_{h_{b-1} h_{b}}, q_{h_{b-1} h_{b}}^{\prime}\right\rangle$ from (6) and by applying Lemma 1 we get the the sequence of inequalities,

$\left\langle q_{i j}^{3 n-2}, q_{i j}^{13 n-2}\right\rangle \leq\left\langle q_{i j}^{3 n-2-p}, q_{i j}^{13 n-2-p}\right\rangle \leq\left\langle q_{i j}^{3 n-2-p+2}, q_{i j}^{13 n-2-p+2}\right\rangle \leq \ldots \leq\left\langle q_{i j}^{3 n-4}, q_{i j}^{13 n-4}\right\rangle$. By applying Lemma 1 while $p \leq n$ and $3 n-2-p \geq n$

Case (ii) Among $\mathrm{p}, \mathrm{q}$ and $\mathrm{s}$ no one is even but sum of any two is even. Three subcases exist

(a) $b \leq c$ and $d \leq e$. Let $b=n$ or $e=2 n-2$, if $b=n$, then $q+s \leq 2 n-2$ and $3 n-2-q-s \geq n$. So if $b<n$ and $e>2 n-2$ then $p+s \leq 2 n-2$. Both cases coincides with case (i). 
(b) ( $b>c$ and $d \leq e$ ) or $(b \leq c$ and $d>e$ ), if $b>c$ and $d \leq e$. Then $b=n, c=n-1$ and $q+s \leq 2 n-2$.

(c) $b>c$ and $d>e$ that is $b=n, c=n-1, d=2 n-1$ and $e=2 n-2$. If $a>0$ or $f<3 n-2$ implies $p+s \leq 2 n-2$. Put $a=0$ and $f=3 n-2$ then $\left\langle q_{h_{n-1} h_{n}}, q_{h_{n-1} h_{n}}^{\prime}\right\rangle=\left\langle q_{k i}, q_{k i}^{\prime}\right\rangle$ and $\left\langle q_{h_{2 n-2} h_{2 n-1}}, q_{h_{2 n-2} h_{2 n-1}}^{\prime}\right\rangle=\left\langle q_{j k}, q_{j k}^{\prime}\right\rangle$ for a few $k \in\{1,2, \ldots, n\}$. If $\left\langle q_{k i}, q_{k i}^{\prime}\right\rangle>\left\langle q_{i k}, q_{i k}^{\prime}\right\rangle$ and $\left\langle q_{j k}, q_{j k}^{\prime}\right\rangle>\left\langle q_{k j}, q_{k j}^{\prime}\right\rangle$ then we will have $\left\langle q_{h_{\alpha} h_{\beta}}, q_{h_{\alpha} h_{\beta}}^{\prime}\right\rangle \leq\left\langle q_{h_{\beta} h_{\alpha}}, q_{h_{\beta} h_{\alpha}}^{\prime}\right\rangle$ for some $\alpha, \beta \in\{n, n+1, n+2, \ldots, 2 n-2\}$ already proved in Lemma 1 . Eradicating

$$
\left\langle q_{h_{0} h_{1}}, q_{h_{0} h_{1}}^{\prime}\right\rangle \wedge\left\langle q_{h_{1} h_{2}}, q_{h_{1} h_{2}}^{\prime}\right\rangle \wedge, \ldots, \wedge\left\langle q_{h_{n-1} h_{n}}, q_{h_{n-1} h_{n}}^{\prime}\right\rangle
$$

and

$$
\left\langle q_{h_{2 n-2} h_{2 n-1}}, q_{h_{2 n-2} h_{2 n-1}}^{\prime}\right\rangle \wedge\left\langle q_{h_{2 n-1} h_{2 n}}, q_{h_{2 n-1} h_{2 n}}^{\prime}\right\rangle \wedge, \ldots, \wedge\left\langle q_{h_{3 n-3} h_{3 n-2}}, q_{h_{3 n-3} h_{3 n-2}}^{\prime}\right\rangle
$$

from (6) put $\left\langle q_{h_{\alpha} h_{\beta}}, q_{h_{\alpha} h_{\beta}}^{\prime}\right\rangle \wedge\left\langle q_{h_{\beta} h_{\alpha}}, q_{h_{\beta} h_{\alpha}}^{\prime}\right\rangle$ into (6) and applying Lemma 1, we obtain the result so, we consider when $\left\langle q_{k i}, q_{k i}^{\prime}\right\rangle \leq\left\langle q_{i k}, q_{i k}^{\prime}\right\rangle$ or $\left\langle q_{j k}, q_{j k}^{\prime}\right\rangle \leq\left\langle q_{k j}, q_{k j}^{\prime}\right\rangle$, say $\left\langle q_{j k}, q_{j k}^{\prime}\right\rangle \leq\left\langle q_{k j}, q_{k j}^{\prime}\right\rangle$. Eradicating

$$
\left\langle q_{h_{0} h_{1}}, q_{h_{0} h_{1}}^{\prime}\right\rangle \wedge\left\langle q_{h_{1} h_{2}}, q_{h_{1} h_{2}}^{\prime}\right\rangle \wedge, \ldots, \wedge\left\langle q_{h_{n-1} h_{n}}, q_{h_{n-1} h_{n}}^{\prime}\right\rangle
$$

and

$$
\left\langle q_{h_{2 n-2} h_{2 n-1}}, q_{h_{2 n-2} h_{2 n-1}}^{\prime}\right\rangle \wedge\left\langle q_{h_{2 n-1} h_{2 n}}, q_{h_{2 n-1} h_{2 n}}^{\prime}\right\rangle \wedge, \ldots, \wedge\left\langle q_{h_{3 n-3} h_{3 n-2}}, q_{h_{3 n-3} h_{3 n-2}}^{\prime}\right\rangle
$$

from (6), put $\left\langle q_{j k}, q_{j k}^{\prime}\right\rangle \wedge\left\langle q_{k j}, q_{k j}^{\prime}\right\rangle$ into (6) and applying Lemma 1 we can write

$$
\begin{aligned}
\left\langle q_{i j}^{3 n-2}, q_{i j}^{\prime 3 n-2}\right\rangle & \leq\left\langle q_{h_{i} h_{n+}}, q_{h_{i} h_{n+1}}^{\prime}\right\rangle \wedge\left\langle q_{h_{n+1} h_{n+2}}, q_{h_{n+1} h_{n+2}}^{\prime}\right\rangle \wedge, \ldots, \wedge\left\langle q_{h_{2 n-3} j}, q_{h_{2 n-3} j}^{\prime}\right\rangle \wedge\left\langle q_{j k}, q_{j k}^{\prime}\right\rangle \wedge\left\langle q_{k j}, q_{k j}^{\prime}\right\rangle \leq\left\langle q_{i j}^{n}, q_{i j}^{\prime n}\right\rangle \\
& \leq\left\langle q_{i j}^{n+2}, q_{i j}^{\prime n+2}\right\rangle \leq \ldots \leq\left\langle q_{i j}^{3 n-4}, q_{i j}^{\prime 3 n-4}\right\rangle .
\end{aligned}
$$

Hence proved.

\section{Example 3.}

$Q_{1}=\left(\begin{array}{l}\langle 0.0,1.0\rangle\langle 0.8,0.2\rangle\langle 0.1,0.8\rangle\langle 0.1,0.8\rangle\langle 0.0,1.0\rangle \\ \langle 0.0,1.0\rangle\langle 0.0,1.0\rangle\langle 0.8,0.2\rangle\langle 0.1,0.8\rangle\langle 0.0,1.0\rangle \\ \langle 0.0,1.0\rangle\langle 0.0,1.0\rangle\langle 0.0,1.0\rangle\langle 0.8,0.2\rangle\langle 0.0,1.0\rangle \\ \langle 0.0,1.0\rangle\langle 0.0,1.0\rangle\langle 0.0,1.0\rangle\langle 0.0,1.0\rangle\langle 0.8,0.1\rangle \\ \langle 0.8,0.2\rangle\langle 0.1,0.8\rangle\langle 0.1,0.8\rangle\langle 0.9,0.1\rangle\langle 0.0,1.0\rangle\end{array}\right) \quad$ and $Q_{2}=\left(\begin{array}{l}\langle 0.0,1.0\rangle\langle 0.2,0.7\rangle\langle 0.0,1.0\rangle\langle 0.0,1.0\rangle\langle 0.0,1.0\rangle \\ \langle 0.0,1.0\rangle\langle 0.0,1.0\rangle\langle 0.3,0.6\rangle\langle 0.0,1.0\rangle\langle 0.0,1.0\rangle \\ \langle 0.0,1.0\rangle\langle 0.0,1.0\rangle\langle 0.0,1.0\rangle\langle 0.4,0.5\rangle\langle 0.0,1.0\rangle \\ \langle 0.0,1.0\rangle\langle 0.0,1.0\rangle\langle 0.0,1.0\rangle\langle 0.0,1.0\rangle\langle 0.5,0.4\rangle \\ \langle 0.8,0.2\rangle\langle 0.1,0.8\rangle\langle 0.0,1.0\rangle\langle 0.0,1.0\rangle\langle 0.0,1.0\rangle\end{array}\right)$

It can be seen that $Q_{1}$ is s-transitive IFM and $Q_{2}$ is not s-transitive by Theorem 6 . Then we have $Q_{1}^{k} \neq Q_{1}^{m}$ for all $k, m \in\{1,2, \ldots 11\}$ and $Q_{2}^{k} \neq Q_{2}^{m}$ for all $k, m \in\{1,2, \ldots 17\} \quad(k \neq m)$.

\section{Canonical form of s-transitivity IFM}

Theorem 9. If $Q$ is s-transitive IFM then there exist a permutation IFM $P$ such that $T=\left(\left\langle t_{i j}, t_{i j}^{\prime}\right\rangle\right)=P \times Q \times P^{T}$ satisfies $\left\langle t_{i j}, t_{i j}^{\prime}\right\rangle \geq\left\langle t_{j i}, t_{j i}^{\prime}\right\rangle$ for $i>j$.

Proof. From Theorem 1 we have $Q=\Delta Q \vee \nabla Q$. Obviously, $\nabla Q$ is symmetric. By Theorem $7 \Delta Q$ is nilpotent since $\mathrm{Q}$ is s-transitive IFM. Thus and by Theorem 2 permutation IFM $P$ exists.

Theorem 9 is the generalization of Theorem 3 which is Lee's generalization of a similar result concerning idempotent fuzzy matrices and introduced by [31]. To construct $\mathrm{P}$ the following procedure should be followed.

(1) $(i, j)^{t h}$ entry of Q must be $(h, k)$ entry of $\mathrm{T}$ iff $\left\langle p_{h i}, p_{h i}^{\prime}\right\rangle=\left\langle p_{k j}, p_{k j}^{\prime}\right\rangle=\langle 1,0\rangle$

(2) The permutation matrix is 


$$
\left\langle q_{i j}, q_{i j}^{\prime}\right\rangle>\left\langle q_{j i}, q_{j i}^{\prime}\right\rangle \Rightarrow\left\langle p_{h i}, p_{h i}^{\prime}\right\rangle=\left\langle p_{k j}, p_{k j}^{\prime}\right\rangle=\langle 1,0\rangle \text { with } h>k
$$

(3) Let $\rho \subset I \times I$, where $I=\{1,2, . . n\}$, and classify as $i \rho j \Leftrightarrow(i, j) \in \rho \Leftrightarrow\left\langle q_{i j}, q_{i j}^{\prime}\right\rangle>\left\langle q_{j i}, q_{j i}^{\prime}\right\rangle$. Clearly $(i, i) \notin \rho$ so P is irreflexive and transitive.

(4) Define $\pi$ such that $\rho \subset \pi$ and $(i, j) \in \pi$ or $(j, i) \in \pi$.

(5) $(p(1), p(2), \ldots, p(n))$ in the linear ordering $\pi$ from minimum to the maximum one.

(6) In the set $U=(p(1), p(2), \ldots, p(n)), p(i)>p(j)$ iff $(i, j) \in \pi$.

(7) By applying (7) and the definitions of $\rho$ and $\pi$ for any $s \in I$,

$$
\left\langle p_{r s}, p_{r s}^{\prime}\right\rangle= \begin{cases}\langle 1,0\rangle & \text { for } q=p(s) \\ \langle 0,1\rangle & \text { for } q \in(U-p(s)) .\end{cases}
$$

\section{Example 4.}

$$
Q=\left(\begin{array}{l}
\langle 0.1,0.7\rangle\langle 0.5,0.4\rangle\langle 0.3,0.6\rangle\langle 0.0,1.0\rangle \\
\langle 0.5,0.4\rangle\langle 0.0,1.0\rangle\langle 0.7,0.2\rangle\langle 0.7,0.2\rangle \\
\langle 0.0,1.0\rangle\langle 0.5,0.4\rangle\langle 0.4,0.5\rangle\langle 0.0,1.0\rangle \\
\langle 1.0,0.0\rangle\langle 0.8,0.1\rangle\langle 0.3,0.6\rangle\langle 1.0,0.0\rangle
\end{array}\right)
$$

Since $\mathrm{Q}$ is strongly transitive IFM but not max-min transitive IFM. So we get numberring $(3,2,1,4)$ corresponding to the relation $\pi_{1}$. Hence by (8), the permutation IFM satisfying the conditions of Theorem 4.1 are following

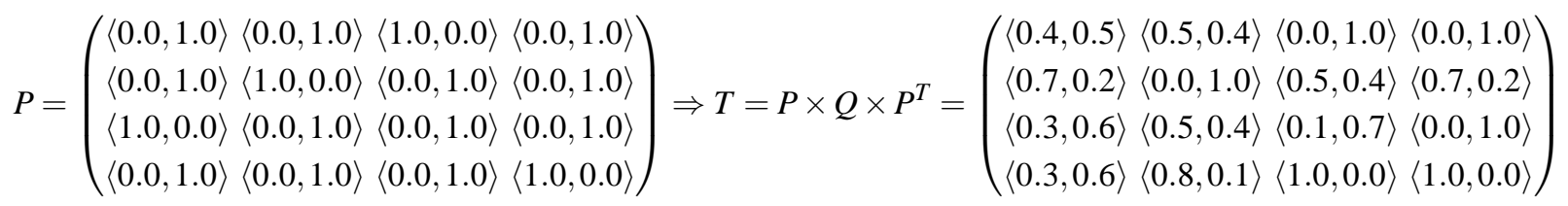

is such that $\left\langle t_{i j}, t_{i j}^{\prime}\right\rangle \geq\left\langle t_{j i}, t_{j i}^{\prime}\right\rangle$ for $i>j$.

\section{Conclusion}

In this article some important properties of s-transitive IFMs are explored. The concept of convergence and canonical form of s-transitive IFMs are discussed and this work is on continuation...

\section{Competing interests}

The authors declare that they have no competing interests.

\section{Authors' contributions}

All authors have contributed to all parts of the article. All authors read and approved the final manuscript.

\section{References}

[1] L.A. Zadeh ., Fuzzy Sets, Journal of Information and Control, 8, (1965). 
[2] K. Atanassov ., Intuitionistic Fuzzy Sets, VII ITKR's Session, Sofia, June (1983).

[3] K. Atanassov ., Intuitionistic Fuzzy Sets ; Theory and Applications, Physica Verlag, (1999).

[4] K. Atanassov., Intuitionistic Fuzzy Implications and Modus Ponens, Notes On Intuitionistic Fuzzy Sets, . 11(1), (2005), 1-5.

[5] K. Atanassov ., On Some Types of Fuzzy Negations, Notes on Intuitionistic Fuzzy Sets 11(4), (2005), 170-172.

[6] K. Atanassov., A New Intuitionistic Fuzzy Implication from a Modal Type, Advance Studies In Contemporary Mathematics 12(1), (2006), 117-122.

[7] K. Atanassov, and G. Gargov., Elements of Intuitionistic Fuzzy Logic.Part I, Fuzzy Sets and Systems, 95(1998),39-52.

[8] Y.B. Im, E. P Lee., The determinant of square intuitionistic fuzzy matrices. Far-East Journal of Mathematical Sciences 5 (2001) 789-796

[9] S.K Khan, M. Pal and A. K. Shyamal.,Intuitionistic Fuzzy Matrices, Notes on Intuitionistic Fuzzy Sets, 8(2) (2002), 51-62.

[10] A. K. Shyamal , M. Pal., Distances between intuitionistics fuzzy matrices. V. U. J. Physical Sciences 8, (2002) 81-91.

[11] M. Bhowmik, M. Pal., Some results on intuitionistic fuzzy matrices and intuitionistic circulant fuzzy matrices. International Journal of Mathematical Sciences 7(1-2), (2008), 177-192.

[12] M. Bhowmik , M. Pal., Generalized intuitionistic fuzzy matrices. Far-East Journal of Mathematical Sciences 29(3), (2008), 533554

[13] A.R. Meenakshi , and T. Gandhimathi., Intuitionistic Fuzzy Relational Equations, Advances in Fuzzy Mathematics, 5 (3), (2010), 239-244.

[14] S. Sriram and P. Murugadas.,On Semi-ring of Intuitionistic Fuzzy Matrices, Applied Mathematical Science, 4(23), (2010), 10991105.

[15] S. Sriram and P. Murugadas., Sub-inversesof Intuitionistic Fuzzy Matrices, Acta Ciencia Indica Mathematics, Vol.XXXVII, M No. 1, (2011), 41-56.

[16] P. Murugadas and K. Lalitha., Dual implication Operator in Intuitionistic Fuzzy Matrices, Int.Conference on Mathematical Modelling and its Applications, Dec 22-24,2012, Organized by Department of Mathematics, Annamalai University.

[17] P. Murugadas and K. Lalitha.,Sub-inverse and g-inverse of an Intuitionistic Fuzzy Matrix Using Bi-implication Operator, Int.Journal of Computer Application, 89(1), (2014), 1-5.

[18] P. Murugadas and K. Lalitha.,Implication Operator on Intuitionistic Fuzzy Tautological Matrix, Int.Journal of Fuzzy Mathematical Archive, 5(2), (2014), 79-87.

[19] P. Murugadas and R. A .Padder ., Reduction of an intuitionistic fuzzy rectangular matrix, Annamalai University Science Journal, 49,(2015) 15-18.

[20] M.G Thomason ; Convergence of powers of a fuzzy matrix, J.Math.Anal.Appl. 57, (1977), 476-480.

[21] J.J Buckley; Note on convergence of powers of a fuzzy matrix ; Fuzzy sets and systems. 121, (2001), 363-364.

[22] Z.T Ran and D.F Liu ; On the oscillating power sequence of a fuzzy matrix, Fuzzy sets and systems. 93, (1998) 75-85.

[23] D.A Gregory, S.Kirkland and N.J Pullman; Power convergent Boolean matrices, Linear algebra and its applications. 179, (1993) 105-117.

[24] H.Hashimoto; Convergence of powers of a fuzzy transitive matrix, Fuzzy sets and systems. 9, (1983),153-160.

[25] Y.Y Lur, Y.K Wu and S.M Guu. Convergence of powers for a fuzzy matrix with convex combination of maxmin and max-arithmetic mean operations, Information Sciences. 179, (2009) 938-944.

[26] W. Kolodziejczyk, Convergence of powers of s-transitive fuzzy matrix, Fuzzy Sets and Systems, 26, (1988) 127-130.

[27] L. J Xin, Convergence of powers of controllable fuzzy matrices, Fuzzy Sets and Systems, 45, (1994) 83-88.

[28] A. D. Nola,Convergence of powers of reciprocal fuzzy matrices, Information Sciences, 75, (1993) 99-107.

[29] W. Kolodziejczyk. Canonical form of a strongly transitive fuzzy matrix, Fuzzy Sets and Systems. 22, (1987), $297-302$.

[30] H. Chenggong. Canonical form of strongly transitive matrices over lattices , Fuzzy Sets and Systems. 45, (1992), $219-222$.

[31] H.Hashimoto. Canonical form of transitive fuzzy matrix, Fuzzy Sets and Systems. 11, (1983), 157-162.

[32] H. Y. Lee and N. G Jeong. Canonical form of a transitive intuitionistic fuzzy matrices, Honam Mathematical Journal. 27(4), (2005), 543-550.

[33] M. Pal and S. K. Khan, Intuitionistic fuzzy matrices, Notes on Intuitionistic Fuzzy Sets, 8(2), (2002), 51-62. 